\title{
Antioxidant activity and phenolic content of phenolic rich fractions obtained from black cumin (Nigella sativa) seedcake.
}

\begin{abstract}
The antioxidant activities of crude methanolic extract (CME) and its fractions using ethyl acetate (EAF), hexane (HF) and water (WF) of black cumin seedcake were investigated. DPPH radical scavenging activity, $\beta$-carotene-linoleate bleaching, and inhibition of corn oil oxidation were used to evaluate the antioxidant capacity. The total phenolics were found to be 78.8, 27.8, 32.1 and 12.1 mg gallic acid equivalents (GAE)/g in EAF, CME, WF and HF, respectively. The CME and EAF exhibited the highest DPPH followed by WF and HF. The extract/fractions showed high effect on reducing the oxidation of $\beta$-carotene. The effect of extract/fractions on the oxidative stability of corn oil at $70{ }^{\circ} \mathrm{C}$ was tested in the dark and compared with butylated hydroxyanisole (BHA). The oil peroxide and anisidine values were generally lower with addition of PRFs in comparison to a control. The predominant phenolic compounds identified by HPLC-DAD in CME and WF of black cumin seedcake were hydroxybenzoic, syringic and p-cumaric acids.
\end{abstract}

Keyword: $\beta$-carotene-linoleic acid assay; 1,1-Diphenyl-2-picrylhydrazyl (DPPH); Antioxidant activity; Nigella sativa; Oil stability; Phenolic rich fractions 